\title{
Rapid Evaluation of the Permeability of Organic-Rich Shale Using the 3D Intermingled-Fractal Model
}

\author{
Caoxiong Li and Mian Lin, Institute of Mechanics, Chinese Academy of Sciences and School of Engineering \\ Science, University of Chinese Academy of Sciences; Lili Ji and Wenbin Jiang, Institute of Mechanics, \\ Chinese Academy of Sciences; and Gaohui Cao, Institute of Mechanics, Chinese Academy of Sciences \\ and School of Engineering Science, University of Chinese Academy of Sciences
}

\begin{abstract}
Summary
Shale possesses abundant micro/nanopores. Most micro/nanopores that exist in organic-rich shale are organic pores and mainly developed in organic matter. The pore distribution in matrix space significantly affects gas percolation and diffusion. Pore-size distribution possesses a self-similar, or fractal, property. The pore space and gas permeability of shale can be easily rebuilt and evaluated, respectively, using fractal theory. In this work, a 3D intermingled-fractal model (3D-IFM) is successfully built using scalable scanning-electron-microscopy (SEM) images of shale samples. 3D-IFM is made up of several components, including organic pores in organic matter and in pyrites, inorganic pores, slits, and matrix. An improved pore-connective-calculation method is also introduced to evaluate the apparent gas permeability of the shale model. The proposed 3D-IFM rapid-permeability-evaluation method for organic-rich shale is valid and useful and considers the main components of shale. This method can rapidly evaluate apparent gas permeability and simplify the apparent-gaspermeability-calculation process. Thus, the method provides a promising means of rapidly evaluating apparent gas permeability.
\end{abstract}

\section{Introduction}

Most hydrocarbon in shale gas is stored in tight matrix blocks that contain an assemblage of rocks, reservoirs, and caprocks. Organicrich shale has an ultralow permeability and porosity compared with those in a normal reservoir. Most pores are at the micro/nanoscale and exist as organic, inorganic, and mineral-matrix pores. Fluid-transport mechanisms in shale are special. Non-Darcy effects, including Knudsen diffusion and slip flow, are significant flow mechanisms for fluid transport in nanopores (Roy et al. 2003; Cooper et al. 2004; Javadpour et al. 2007). The non-Darcy effects can significantly enhance apparent permeability for shale gas (Javadpour 2009; Shabro et al. 2009, 2014). Neglecting non-Darcy effects in shale will evidently underestimate apparent permeability. Because of the complex flow mechanism in shale, investigation of the gas-transport mechanism and rapid evaluation of permeability in organic-rich shale are important for the analysis of gas production, hydraulic fracturing, and production forecasting.

Shale has a relatively complex pore space with various components, such as organic pores in organic matter, organic pores in pyrites, inorganic pores within clay platelets, and clay aggregates. The connectivity of pores significantly affects the shale-gas-exploration process. Precisely rebuilding the pore distribution and pore shape in 3D space is difficult. Simplification of the rebuilding process is necessary in the permeability-calculation process. Many studies have adopted the rebuilding method for pore spaces. Theoretical pore space has developed from the parallel-tube-bundle model to the network model. Fatt (1956) introduced the parallel-tube-bundle model to represent pore space depending on the actual pore-size distribution of a network model. Fischer and Celia (1999) investigated the relationship between confined pressure and relative permeability by introducing the capillary pressure curve into the pore-network model. Bryant and Blunt (1992) and Bryant et al. (1993) studied the influence of confined pressure on permeability depending on the stress deformation of the pore-network model. Jiang et al. (2017) studied gas transport in pore space using a high-precision porenetwork-extraction algorithm. Shabro et al. (2012) investigated the influence of diffusion, slip, and adsorption/desorption effect on gaspermeability models. Yuan et al. (2017) and Feng and Gray (2017) studied the introduced fractures and effect of fractures for shale-play production in both mechanism and application. Lin et al. (2017) summarized several fractal gas-permeability models. Niu et al. (2014) studied second-order gas-permeability correlation for slip flow in shale. Wu et al. (2016a, b) derived a unified model for gas transfer in nanopores of shale-gas reservoirs.

A fractal (deterministic fractal) is a geometric figure for which each and every part is similar to the whole, and which is repeated on different scales (Pia and Sanna 2013a). It is widely accepted that the porosity and fractures in geological formations exhibit fractal features over many length scales (Balankin and Elizarraraz 2012a). The pores in shale also possess fractal properties at a certain scale (Thompson et al. 1987; Thovert et al. 1990; Adler 1996; Adler and Thovert 1998). Fractal theory is a helpful and convenient tool for representing pore space and evaluating permeability using self-similar properties (Yu and Li 2001; Yu 2005). Yu et al. (2002) developed a fractal in-plane permeability model for various fabrics. Xu and Yu (2008) developed a permeability-prediction model for homogeneous porous media by introducing a cross-sectional parameter. Cai et al. (2010) and Cai (2014) established an effective-permeability model depending on spontaneous imbibition and used the model in several applications, and then introduced the low-velocity non-Darcy term for low-permeability porous media. Shou et al. (2010) developed a difference-fractal model for predicting the permeability of fibrous porous media. Xiao et al. (2017) and Lu et al. (2018) investigated multiphase flow in fractal porous media. Wei et al. (2015) used numerical simulation to study transport properties in fractal porous media. Balankin and Elizarraraz (2012a, b) developed a fractal continuum model to investigate fractal continuum hydrodynamics and related applications to model fluid flows in fractally permeable reservoirs. Moreover, the fractal continuum model was later developed to simulate stresses, strains, and other characteristics for fractal porous media (Balankin 2013, 2015a, b). Perfect (2005) and Perfect et al. (2006) developed another method to calculate liquid flow in multifractal porous media, combining effective hydraulic conductivity and geometrical multifractals. This method has special advantages in the upscaling process for fractal porous media. Cihan et al. (2009a) calculated the permeability of a matrix with 3D random pore distribution using the probabilistic-capillary-connectivity (PCC) method. The PCC method is a novel and effective way to

Copyright $@ 2018$ Society of Petroleum Engineers

Original SPE manuscript received for review 30 January 2018. Revised manuscript received for review 15 March 2018. Paper (SPE 191358) peer approved 22 March 2018. 
calculate permeability for the random fractal model, especially the 3D model. The PCC method has been widely developed in multiphase flow, including predicting relative permeability (Cihan et al. 2009b), the capillary pressure curve (Cihan et al. 2014), and the saturation distribution of each phase (Cihan et al. 2017).

Pia and Sanna (2013a) built an intermingled-fractal-unit (IFU) method to simplify the pore space of porous media. The IFU, combined by several different fractal units of the "Sierpinski carpet" type, is an effective way to reconstruct fractal and nonfractal pore space. The key of this method is to follow the pore cumulative curve for real porous media by adjusting the fractal parameters of the IFU model. This method is used not only for fluid-flow description in porous media, but also for thermal conductivity (Pia and Sanna 2013a), electrical conductivity (Pia and Sanna 2013b), permeability (Pia and Sanna 2014; Pia 2016), and other characteristics (Pia et al. 2016). The IFU method greatly simplified the expression process of complicated porous media. Li et al. (2017) improved the IFU method to investigate the transport characteristics of shale.

\section{Basic Theory of the Fractal Model and Permeability Calculation}

Introduction of the 3D-IFU Model. The IFM is a useful tool for representing pores in porous media. Pia and Sanna (2013a) developed a fractal method called the IFU model to represent the microstructure of voids in pores. This model intermingles several types of fractal units. Each unit is a revised version of the Sierpinski carpet model. The volume (area for plane) of the IFU model can expressed as

$$
V_{\text {res }}=n_{A} V_{A}+n_{B} V_{B}+n_{C} V_{C}
$$

where $V_{\text {res }}$ is the volume/area of the IFU model; $n_{A}, n_{B}$, and $n_{C}$ are unit numbers; and $V_{A}, V_{B}$, and $V_{C}$ are the volume/area of the units.

The void space for the IFU model contains several subunits, typically two or three subunits, according to Pia and Sanna (2013a). For easy understanding, we assumed a typical IFU model combined with Units A, B, and C, with each unit possessing different iteration parameters. To show the basic structure of the units (e.g., Unit A) with different iteration parameters, we illustrated typical 2D and 3D fractal models in Fig. 1. In Figs. 1a, 1b, and 1c, the white area is void space, the black area is the iteration area, and the shaded area is a noniteration space. Iteration factor $F$ is the number of generated squares on each side in one iteration process. During each iteration, the sides of a square are divided by three $(F=3)$, thus obtaining nine subsquares (for a plane) with several squares removed. Fig. 1a shows that three squares are removed. In the next iteration, each remaining subsquare is regarded as a new square and divided into nine subsquares, and the same numbers of subsquares are removed. For a noniteration block, the block is not considered in the iteration process. $N_{p}$ represents the number of squares removed in each iteration. $N_{\text {solid }}$ represents the number of squares that remain solid in each iteration. $\lambda_{i}$ represents the edge length of the void area in the $i$ th iteration, and $i$ represents the iteration number. $D_{F}$ is the fractal dimension of each unit. In Unit $\mathrm{A}$, these parameters can be expressed as $F_{A}, N_{A p}, N_{A s o l i d}, \lambda_{A i}$, and $i_{A}$. The rest of the parameters in Units B and C can be expressed in the same manner but with different fractal parameters.

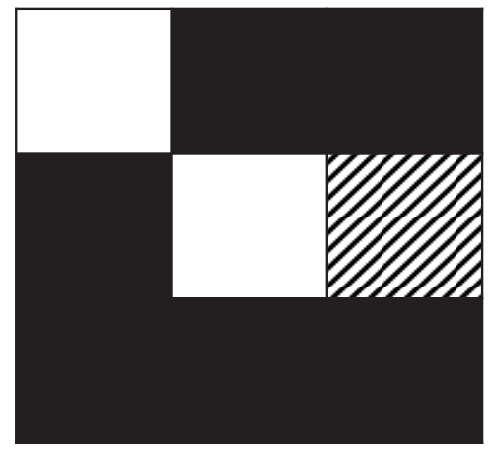

(a) $i=1$

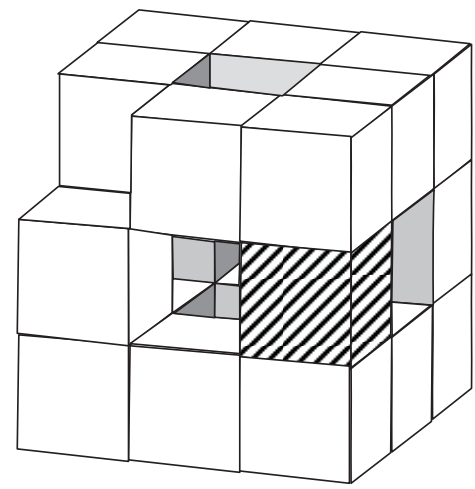

(d) $i=1$

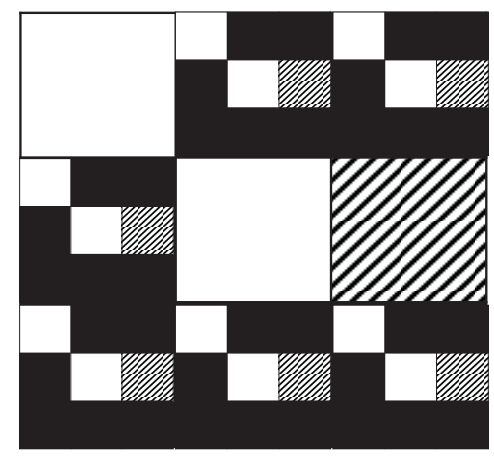

(b) $i=2$

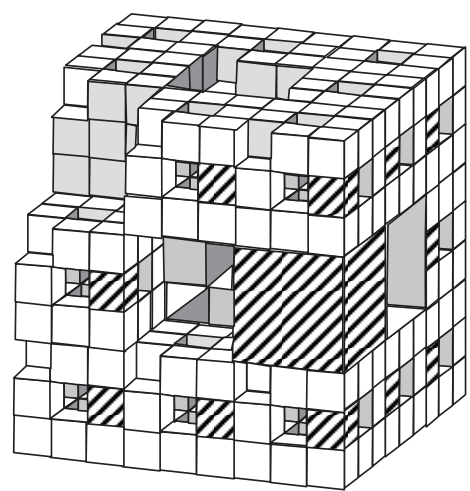

(e) $i=2$

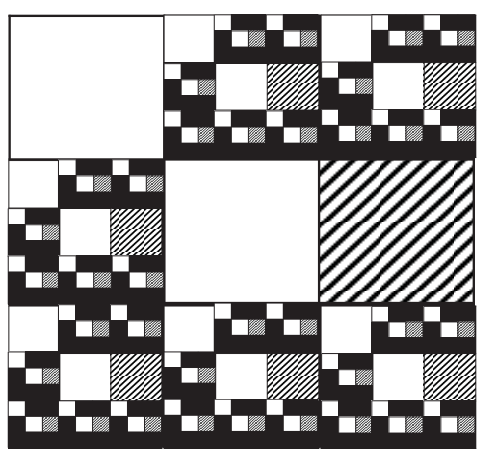

(c) $i=3$

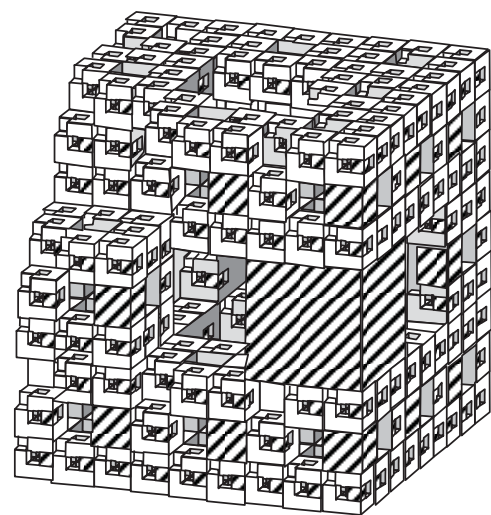

(f) $i=3$

Fig. 1-Schematic of the iteration process in 2D and 3D basic units with different iteration parameters.

IFUs can also be introduced to the 3D space, as shown in Figs. 1d through 1f. In the 3D-IFU model, with $F=3$ as an example, the block can be divided into 27 subblocks. Eight blocks are removed in each iteration. In the next iteration, each of the remaining subsquares is also divided into 27 subblocks with eight blocks removed. A noniteration block is not involved in the iteration process and is marked with slashed lines. 
$\lambda_{A i}=\lambda_{A \max } / F_{A}{ }^{\left(i_{A}-1\right)}$.

The number of void areas generated in the $i_{A}$ th iteration is

$N_{A i}=\left(F_{A}^{2}-N_{A s o l i d}-N_{A p}\right)^{i_{A}-1} \times N_{A p}$

For 3D IFU, the number of void areas is

$N_{A i}=\left(F_{A}^{3}-N_{A s o l i d}-N_{A p}\right)^{i_{A}-1} \times N_{A p}$

where $N_{A \text { solid }}$ is the number of squares that always remain solid. The volume/area of the void space with edge length $\lambda_{A i}$ is

$$
V_{A i}=\lambda_{A i}^{2} N_{A i}
$$

For 3D IFU,

$V_{A i}=\lambda_{A i}^{3} N_{A i}$.

The total volume/area of the void space in Unit A is

$$
V_{A}=V_{A 1}+V_{A 2}+\ldots+V_{A k}+\ldots+V_{A i}=\sum_{k=1}^{i} V_{A k}
$$

where the total volume/area of the void space before the $k$ th iteration is

$$
V_{A k_{\_} \text {accumulate }}=V_{A 1}+V_{A 2}+\ldots+V_{A k} .
$$

Eqs. 1 through 8 are all for one fractal unit, Unit A. An IFM can contain more than one fractal unit, according to Pia and Sanna (2013a); in addition, the pores in the IFU model can be randomized.

Permeability Calculation. The apparent-gas-permeability-calculation method using the 3D intermingled-fractal distribution is discussed in this subsection. In the conventional penetrating system, pores mainly exist at the micron size, and Darcy permeability is commonly used. However, for nanopores, Darcy permeability is no longer suitable. For shale-gas flow in nanoscale pores, the influence of noncontinuum effects and dominant surface interactive forces emerged (Javadpour et al. 2007). Generally, there are four regimes for gas flows: continuum, slip, transition, and free molecular flows, depending on the Knudsen number. Javadpour and Ettehadtavakkol (2015) showed that continuum no-slip flow or the Darcy equation is valid for $K_{n}<10^{-3}$. Continuum flow with slip correction (Klinkenberg) is valid for $K_{n}<10^{-1}$, which covers most conventional gas reservoirs and many tight-gas-reservoir conditions as well. However, because of the existence of nanopores in a shale system, $K_{n}$ could be larger than 0.1 , and hence new forms of gas-flow equations are needed. For a single capillary, Javadpour et al. (2007) provided a permeability equation that considers slip and the Knudsen-diffusion effect as follows:

$$
q_{\text {square }}=\left\{\frac{\lambda \mu M}{3 \times 10^{3} R T \rho_{\text {avg }}}\left(\frac{8 R T}{\pi M}\right)^{0.5}+\frac{\lambda^{2}}{32}\left[1+\left(\frac{8 \pi R T}{M}\right)^{0.5} \frac{2 \mu}{p_{\text {avg }} \lambda}\left(\frac{2}{\alpha}-1\right)\right]\right\} \frac{A}{\mu} \frac{\left(P_{\text {in }}-P_{\text {out }}\right)}{L},
$$

where $\lambda$ is the equivalent tube diameter (for a square tube, $\lambda$ is the edge length); $M$ is the molar mass of natural gas (in $\mathrm{kg} / \mathrm{kmol}$ ); $\mu$ is the viscosity of natural gas (in $\mathrm{Pa} \cdot \mathrm{s}$ ); $R$ is the universal gas constant (in $\mathrm{J} / \mathrm{mol} \cdot \mathrm{K}$ ); $T$ is temperature (in $\mathrm{K}$ ); $\rho_{\text {avg }}$ is the average density of natural gas (in $\mathrm{kg} / \mathrm{m}^{3}$ ); $P_{\text {in }}$ and $P_{\text {out }}$ are inlet and outlet pressures (in Pa), respectively; $p_{\text {avg }}$ is the average gas pressure (in Pa); and $\alpha$ is the tangential-momentum-accommodation coefficient. The viscosity and density of natural gas change significantly with the average pressure; the equations for the viscosity and density of natural gas were tested by Lee et al. (1966) in a laboratory experiment. $A$ is cross-sectional area (in a single tube, $A=\lambda^{2}$ ) and $L$ is the length of the tube. As for adsorption, the model aims at the condition with relatively low average pore pressure. The average pore pressure in our working condition is relatively low (0.55 MPa), and the influence on apparent permeability is negligible under this condition. To simplify the fractal model and the calculation process, the adsorption effect is not included in Eq. 9.

In addition, our focus for this model is to link the PCC with shale permeability considering several ingredients. Certain simplifications are made (Cihan et al. 2009a) to simplify the problem:

- Pores and bulks are simplified as squares/necks corrected by shape factors on the cross section.

- Permeability is related to connecting probability in fractal porous media.

- The distribution for pores and bulks in 3D space is random.

- The number of pores is such that any cross section shares the same fractal dimension and pore-size distribution.

To calculate the 3D-IFU permeability, we introduced the PCC method, which was first widely used by Cihan et al. (2008, 2009b). In the PCC method, as shown in Fig. 2, the connecting probabilities for pores in every cross section are assumed to be equal. Permeability has a positive correlation with the cross section of tubes. When pores are large, the connecting probability is also large; thus, the permeability is large. Additionally, two steps are implemented when calculating the permeability of porous media with the PCC method. The first is to calculate the pore-connective probability; the second is to derive the permeability of porous media using the connective probability and tube-permeability theory. A detailed theoretical explication was provided by Marshall (1958) and Cihan et al. (2009a). With sufficient pores in porous media, we suggest that any cross section shares the same fractal dimension and pore-size distribution. Any two adjacent surfaces are connected through pore throats. The key task is to calculate the connective probability between pairs of 
contacting pores. Void sizes that vary in space are initially sorted according to equivalent diameters in a descending order (Fig. 2). To provide an example, the iteration level, equivalent diameter, number of pores, and probability are listed in Table 1, with a descending order of equivalent diameters. The largest pore is $\lambda_{1}$, which can connect any pores on other surfaces (as an example, $\lambda_{1}$ in Face A can connect every pore in Face B, as shown in Fig. 2). The probability of $\lambda_{1}$ pores in one surface (Face A) connecting to other $\lambda_{1}$ pores in another surface (Face B) is $\left(\lambda_{1}^{2} N_{1} / A\right)^{2}$, shown by the black arrow in Fig. 2. For the second level, the probability of $\lambda_{2}$ pores in Face $\mathrm{A}$ connecting to $\lambda_{1}$ pores in Face B is $\left(\lambda_{1}^{2} N_{1} / A\right) \times\left(\lambda_{2}^{2} N_{2} / A\right)$, shown by the red arrow, and the probability of $\lambda_{2}$ pores in Face A connecting to $\lambda_{2}$ pores in Face B is $\left(\lambda_{2}^{2} N_{2} / A\right)^{2}$, shown by the blue arrow. In addition, the probability of $\lambda_{2}$ pores in Face B connecting to $\lambda_{1}$ pores in Face $\mathrm{A}$ is $\left(\lambda_{1}^{2} N_{1} / A\right) \times\left(\lambda_{2}^{2} N_{2} / A\right)$. We have to obtain the probability $\left(\lambda_{2}^{2} N_{2} / A\right)^{2}+2\left(\lambda_{1}^{2} N_{1} / A\right)\left(\lambda_{2}^{2} N_{2} / A\right)$, as well as that for the following pores in the remaining iteration levels. Each connecting probability is shown in Table 1.
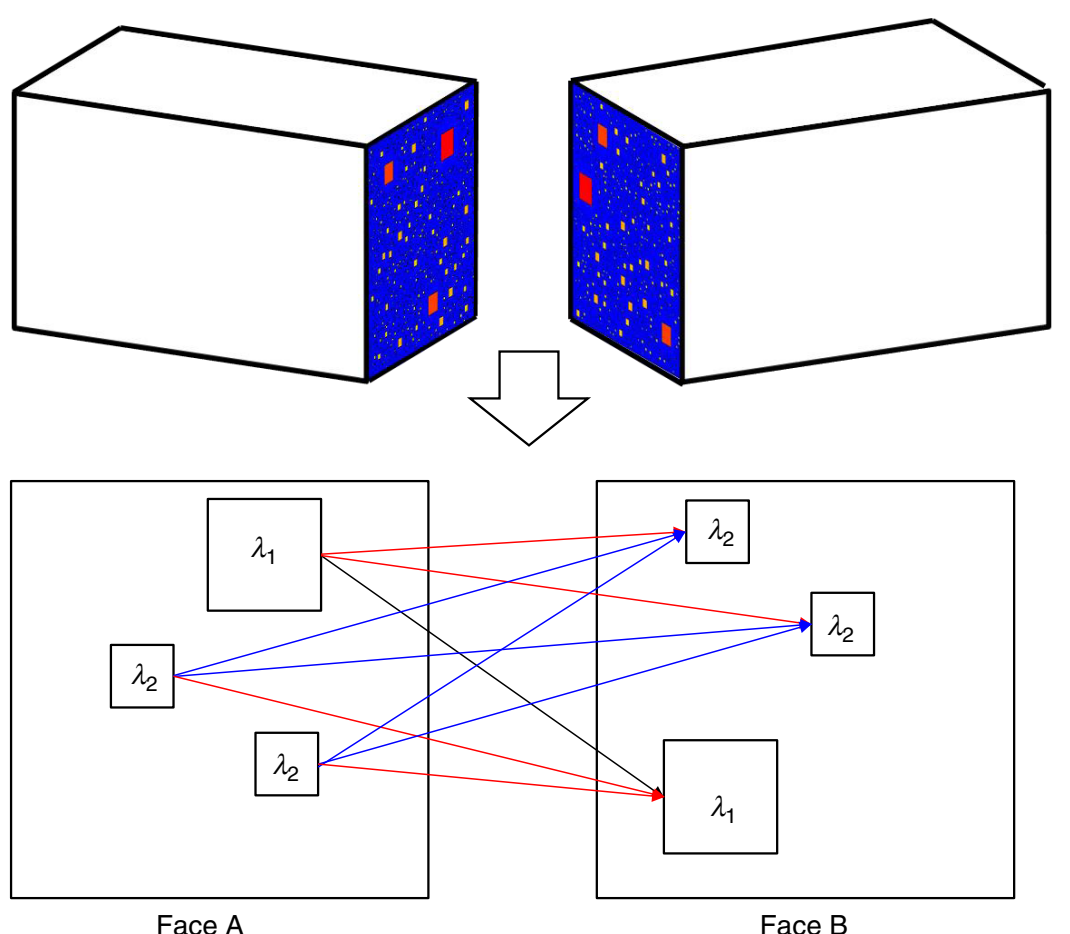

Face A

Face B

Fig. 2-Schematic of pore-connective-calculation method.

\begin{tabular}{cccc} 
Iteration Level $(i)$ & Pore Diameter & Number of Pores & Probability $(P)$ \\
\hline 1 & $\lambda_{1}$ & $N_{1}$ & $\left(\lambda_{1}^{2} N_{1} / A\right)^{2}$ \\
2 & $\lambda_{2}$ & $N_{2}$ & $\left(\lambda_{2}^{2} N_{2} / A\right)^{2}+2\left(\lambda_{1}^{2} N_{1} / A\right)\left(\lambda_{2}^{2} N_{2} / A\right)$ \\
3 & $\lambda_{3}$ & $N_{3}$ & $\left(\lambda_{3}^{2} N_{3} / A\right)^{2}+2\left(\lambda_{1}^{2} N_{1} / A\right)\left(\lambda_{3}^{2} N_{3} / A\right)+2\left(\lambda_{2}^{2} N_{2} / A\right)\left(\lambda_{3}^{2} N_{3} / A\right)$ \\
$\ldots$ & $\ldots$ & $\ldots$ & $\ldots$ \\
\hline
\end{tabular}

Table 1-Connecting-probability-calculation process.

According to Cihan et al. (2009), for the normal Darcy method, permeability is calculated as

$$
k_{D}(n)=C\left[\frac{\lambda_{1}^{2} N_{1}}{A} \frac{\lambda_{1}^{2} N_{1}}{A} \lambda_{1}^{2}+\left(\frac{\lambda_{2}^{2} N_{2}}{A} \frac{\lambda_{2}^{2} N_{2}}{A}+2 \times \frac{\lambda_{1}^{2} N_{1}}{A} \frac{\lambda_{2}^{2} N_{2}}{A}\right) \lambda_{2}^{2}+\left(\frac{\lambda_{3}^{2} N_{3}}{A} \frac{\lambda_{3}^{2} N_{3}}{A}+2 \times \frac{\lambda_{1}^{2} N_{1}}{A} \frac{\lambda_{3}^{2} N_{3}}{A}+2 \times \frac{\lambda_{2}^{2} N_{2}}{A} \frac{\lambda_{3}^{2} N_{3}}{A}\right) \lambda_{3}^{2}+\ldots\right]
$$

where $C$ is the shape factor, determined by Veltzke and Thöming (2012) and Cai et al. (2014). Considering diffusion and the slip effect, we combined the PCC method and the Javadpour method (Javadpour et al. 2007) to calculate the apparent gas permeability for every iteration level in the 3D-IFU model.

For iteration level $i=1$,

$$
k_{n D}(1)=32 C \frac{\lambda_{1}^{2} N_{1}}{A} \frac{\lambda_{1}^{2} N_{1}}{A}\left\{\frac{\lambda_{1} \mu M}{3 \times 10^{3} R T \rho_{\mathrm{avg}}}\left(\frac{8 R T}{\pi M}\right)^{0.5}+\frac{\lambda_{1}^{2}}{32}\left[1+\left(\frac{8 \pi R T}{M}\right)^{0.5} \frac{2 \mu}{p_{\mathrm{avg}} \lambda_{1}}\left(\frac{2}{\alpha}-1\right)\right]\right\} .
$$


For iteration level $i=2$,

$$
\begin{aligned}
k_{n D}(2)= & 32 C \frac{\lambda_{2}^{2} N_{2}}{A} \frac{\lambda_{2}^{2} N_{2}}{A}\left\{\frac{\lambda_{2} \mu M}{3 \times 10^{3} R T \rho_{\mathrm{avg}}}\left(\frac{8 R T}{\pi M}\right)^{0.5}+\frac{\lambda_{2}^{2}}{32}\left[1+\left(\frac{8 \pi R T}{M}\right)^{0.5} \frac{2 \mu}{p_{\text {avg }} \lambda_{2}}\left(\frac{2}{\alpha}-1\right)\right]\right\} \\
& +2 \times 32 C \frac{\lambda_{1}^{2} N_{1}}{A} \frac{\lambda_{2}^{2} N_{2}}{A}\left\{\frac{\lambda_{2} \mu M}{3 \times 10^{3} R T \rho_{\text {avg }}}\left(\frac{8 R T}{\pi M}\right)^{0.5}+\frac{\lambda_{2}^{2}}{32}\left[1+\left(\frac{8 \pi R T}{M}\right)^{0.5} \frac{2 \mu}{p_{\text {avg }} \lambda_{2}}\left(\frac{2}{\alpha}-1\right)\right]\right\} .
\end{aligned}
$$

For iteration level $i=3$,

$$
\begin{aligned}
k_{n D}(3)= & \left(32 C \frac{\lambda_{3}^{2} N_{3}}{A} \frac{\lambda_{3}^{2} N_{3}}{A}+2 \times 32 C \frac{\lambda_{1}^{2} N_{1}}{A} \frac{\lambda_{3}^{2} N_{3}}{A}+2 \times 32 C \frac{\lambda_{1}^{2} N_{1}}{A} \frac{\lambda_{3}^{2} N_{3}}{A}\right) \\
& \times\left\{\frac{\lambda_{3} \mu M}{3 \times 10^{3} R T \rho_{\mathrm{avg}}}\left(\frac{8 R T}{\pi M}\right)^{0.5}+\frac{\lambda_{3}^{2}}{32}\left[1+\left(\frac{8 \pi R T}{M}\right)^{0.5} \frac{2 \mu}{p_{\mathrm{avg}} \lambda_{3}}\left(\frac{2}{\alpha}-1\right)\right]\right\} .
\end{aligned}
$$

For iteration level $i=n$,

$$
\begin{aligned}
k_{n D}(n)= & \left(32 C \frac{\lambda_{n}^{2} N_{n}}{A} \frac{\lambda_{n}^{2} N_{n}}{A}+2 \times 32 C \frac{\lambda_{1}^{2} N_{1}}{A} \frac{\lambda_{n}^{2} N_{n}}{A}+\ldots 2 \times 32 C \frac{\lambda_{n-1}^{2} N_{n-1}}{A} \frac{\lambda_{n}^{2} N_{n}}{A}\right) \\
& \times\left\{\frac{\lambda_{n} \mu M}{3 \times 10^{3} R T \rho_{\text {avg }}}\left(\frac{8 R T}{\pi M}\right)^{0.5}+\frac{\lambda_{n}^{2}}{32}\left[1+\left(\frac{8 \pi R T}{M}\right)^{0.5} \frac{2 \mu}{p_{\text {avg }} \lambda_{n}}\left(\frac{2}{\alpha}-1\right)\right]\right\} .
\end{aligned}
$$

To sum up, the permeability for a single $3 \mathrm{D}-\mathrm{IFU}$ is

$$
k_{\text {sum- } n D}(n)=\sum_{i=1}^{n} k_{n D}(i) \text {. }
$$

For an IFM combined with several 3D IFUs, the permeability-calculation method is similar to the method listed previously (Eqs. 10-15). The difference is that all pores in each 3D IFU should be listed first in a descending order of equivalent diameters.

\section{Building the IFM}

Basic Information of Samples. A 3D IFM for real samples was built using scalable SEM images. Information for the main components was obtained and shown in Fig. 3. First, the SEM images were obtained; each component is in different resolution. Second, the fractal parameters were derived using the SEM images. Third, 3D IFUs for organic pores, inorganic pores, and organic bulk were built using the fractal parameters. Finally, by intermingling the 3D IFUs, slits, and matrix, we obtained the 3D IFM. The permeability of this model was then calculated and verified.

Samples were obtained from the Longmaxi Marine Shale Formation in Lower Silurian in the Sichuan Basin. Samples 1 and 2 were cut from fresh outcrop in the Qiliao Region of the Longmaxi Formation, and Samples 3 and 4 were twin plugs cut from fresh outcrop in the Shizhu Region of the Longmaxi Formation. The permeability of gas in the dry samples was measured through pulse-decay permeability measurement at $25^{\circ} \mathrm{C}$. Helium is used for samples during the gas permeability test. The pressure difference between the two ends of samples is $1.1 \mathrm{MPa}$. Average temperature and pressure during the permeability test are $306.24 \mathrm{~K}$ and $0.55 \mathrm{MPa}$, respectively. Confining pressures are 3,5,7, and $9 \mathrm{MPa}$. The average gas permeability of each sample was calculated using the quadratic polynomial method to diminish the effect of confining pressure. The experiment results are listed in the Model Validation subsection. The viscosity, density, and compressibility factor of gas changed with the average temperature and pressure. We used the experimental results of Lee et al. (1966).

Scalable SEM was used to analyze the types of pores and the pore-size distribution of the shale samples. Argon ion polishing technology was used for the surface of the samples. The scanning area was $380 \times 380 \mu \mathrm{m}$, with a maximum resolution of $2.5 \mathrm{~nm}$. Through the SEM images, the components of the shale samples were identified as follows: organic pores in organic matter, organic pores in pyrite, distribution of organic bulks and pyrites, inorganic pores, and slits. The different fractal parameters for each component can be derived from different image scales. In addition, the matrix was composed of several components. The mineralogy of the samples was analyzed through energy-dispersive spectroscopy in an area of $400 \times 400 \mu \mathrm{m}$ using a Zeiss Merlin rock-mineralogy-analysis machine with a resolution of $5 \mathrm{~nm}$.

Organic Pores in Organic Matter and Pyrites. Most micro/nanoscale pores in organic-rich shale are organic. Organic pores mainly existed in organic matter and sometimes in pyrites. The first process involved building a fractal model for organic pores. The detailed modeling process is shown in Fig. 4.

1. The organic pores in the organic matter were observed through scalable SEM. The representative elementary surface (RES) for organic pores in organic matter should be calculated because of the heterogeneity of the pore distribution. In this example, we start from the four corners of an image, then expand the target area from the source point (1 pixel on the edge) in the original SEM image and calculate the average grayscale in the subsquare. The variation in the average grayscale stabilized gradually as the pixels on the edge of the subsquare increased. Fig. 4 shows that when the number of pixels on the edge was larger than 480 , the average grayscale remained stable. Thus, the 480 pixels on the edge can be regarded as the RES.

2. In the obtained RES, the grayscale image was segmented to gain organic pores. Threshold value is an important parameter, and the watershed algorithm is helpful in the segmentation process. After segmentation, the grayscale image became a binarized image. The pore cumulative distribution curve of the samples was then derived.

3. We built a 3D-IFU model to express the organic pores in organic matter. The fractal parameters were adjusted to narrow the differences between the cumulative curves of the basic IFU model and the image calculation. Finally, certain parameters of the 3D-IFU model were determined, as shown in Table 2. 


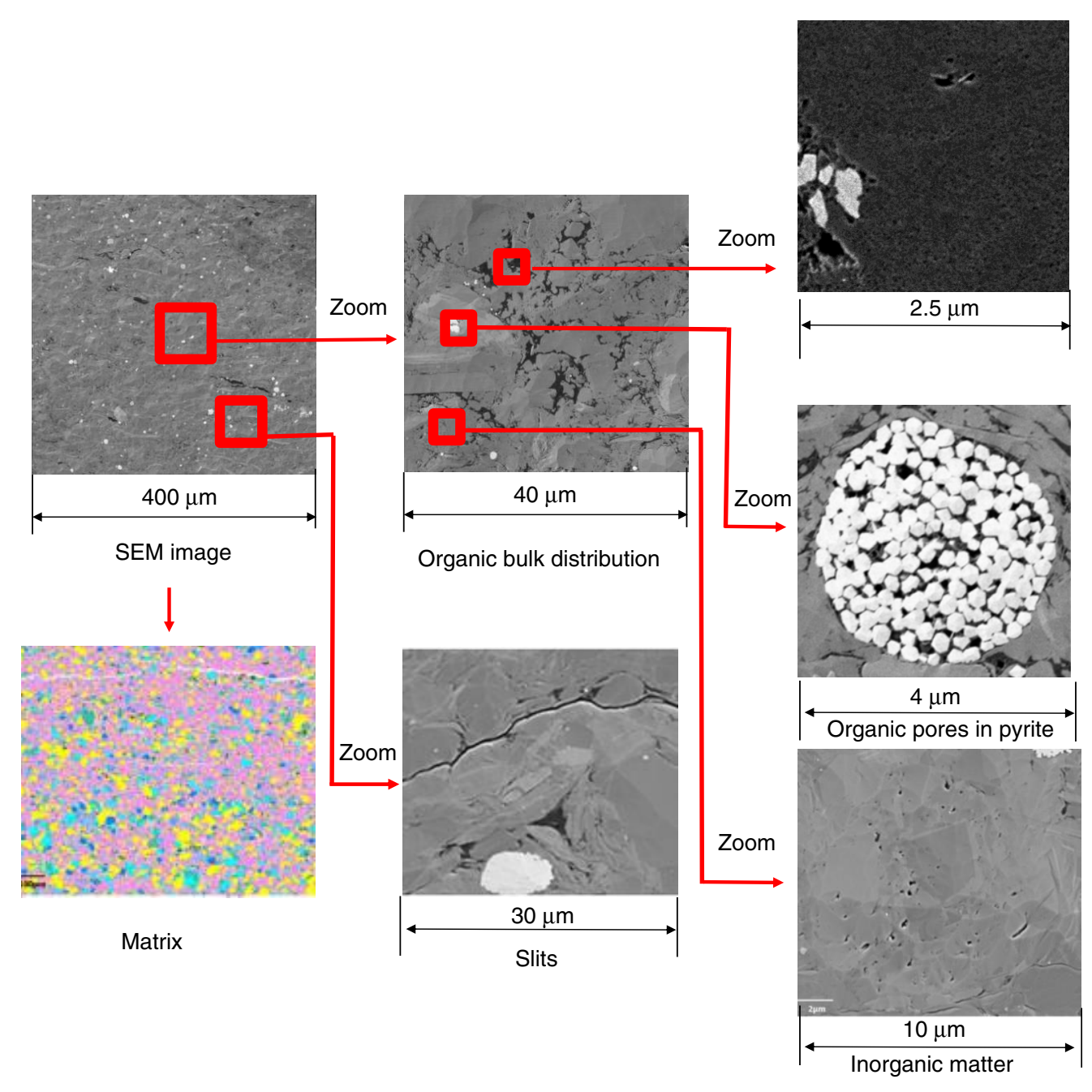

Fig. 3-SEM image of Longmaxi Marine Shale.
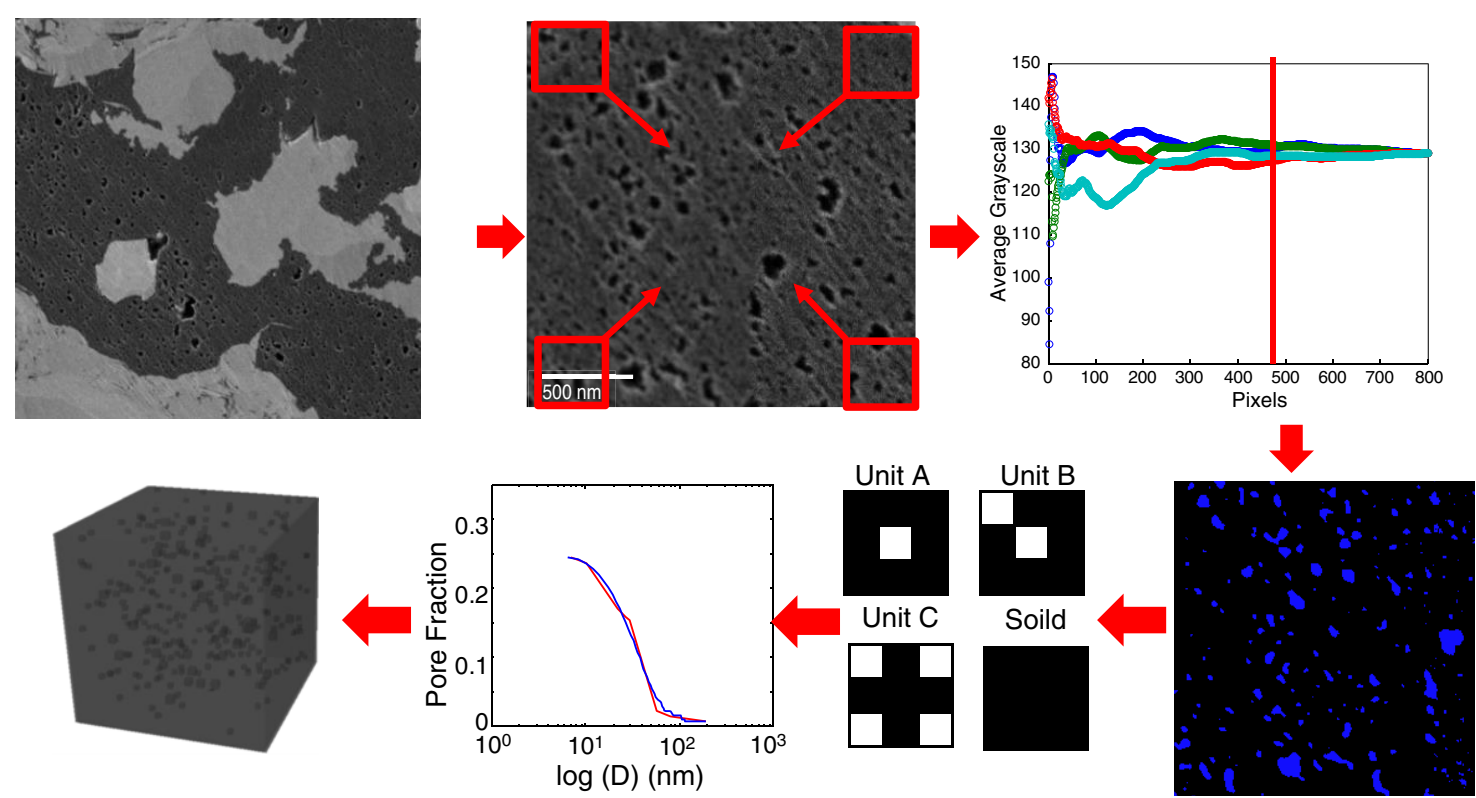

Fig. 4-Modeling process of organic matter.

Organic pores also existed in the pyrites. The pyrites were scattered in the SEM area. Some pyrites possessed organic pores (the overdeveloped pyrites had no pores and can thus be ignored). First, the distribution density of pore-developed pyrites (number of pyrites in this area) was observed from statistical data using the SEM image. Second, the pore-size distribution in a typical pyrite was derived, the fractal parameters were calculated using the matching method on cumulative curves, and the 3D IFU for pyrites was built. Finally, 
the 3D IFUs for pyrites were scattered in the final 3D IFM with an observed distribution density. The building process of the model for the pyrite part is shown in Fig. 5.

1. All the pore-developed pyrites were extracted at a relatively large scale from the SEM image, and the distribution density of pyrites was derived from statistical data. Overdeveloped pyrites were disregarded, suggesting that $N$ pyrites were assumed in a $380 \times 380-\mu \mathrm{m}$ area.

2. The SEM image of a typical pyrite was selected, and some segmentation and binarization were performed on this image. The cumulative pore-size distribution was then calculated.

3. Similar to the building procedure for organic pores in organic matter, the fractal parameters in the pyrite model were adjusted to narrow the differences between the cumulative curves of the IFU model and the image-calculation result. Using the fractal parameters, we obtained the 3D-IFU model for pyrites. The results are shown in Table 3.

4. The 3D-IFU pyrites were then scattered in the final 3D-IFM space depending on the scattered density observed in large-scale images.

\begin{tabular}{|c|c|c|c|c|c|c|c|c|c|c|c|c|}
\hline & \multicolumn{3}{|c|}{ Sample 1} & \multicolumn{3}{|c|}{ Sample 2} & \multicolumn{3}{|c|}{ Sample 3} & \multicolumn{3}{|c|}{ Sample 4} \\
\hline & Unit A & Unit B & Unit C & Unit A & Unit B & Unit C & Unit A & Unit B & Unit C & Unit A & Unit B & Unit C \\
\hline$D_{F}$ & 1.89 & 1.77 & 1.46 & 1.89 & 1.77 & 1.63 & 1.89 & 1.77 & 1.46 & 1.89 & 1.77 & 1.63 \\
\hline$n$ unit & 1 & 6 & 10 & 1 & 4 & 8 & 1 & 5 & 230 & 1 & 23 & 20 \\
\hline$n$ pore $(i=1)$ & 1 & 2 & 4 & 1 & 2 & 3 & 1 & 2 & 4 & 1 & 2 & 3 \\
\hline$\lambda_{\max }(\mathrm{nm})$ & 310 & 180 & 120 & 190 & 100 & 50 & 191 & 80 & 26.67 & 170 & 70 & 50 \\
\hline Iteration & 4 & 4 & 3 & 3 & 3 & 2 & 4 & 3 & 2 & 4 & 3 & 2 \\
\hline$\lambda_{\min }(\mathrm{nm})$ & 11.48 & 6.67 & 13.33 & 21.11 & 11.11 & 16.67 & 7.07 & 8.89 & 8.89 & 6.30 & 7.78 & 16.67 \\
\hline$N_{\text {solid }}$ & 0 & 0 & 0 & 0 & 0 & 0 & 0 & 0 & 0 & 0 & 0 & 0 \\
\hline Total surface $\left(\mu \mathrm{m}^{2}\right)$ & \multicolumn{3}{|c|}{18.25} & \multicolumn{3}{|c|}{7.26} & \multicolumn{3}{|c|}{4.33} & \multicolumn{3}{|c|}{3.35} \\
\hline$\varepsilon_{S E M} / \varepsilon_{I F U}$ & \multicolumn{3}{|c|}{$0.33 / 0.33$} & \multicolumn{3}{|c|}{$0.24 / 0.24$} & \multicolumn{3}{|c|}{$0.30 / 0.30$} & \multicolumn{3}{|c|}{$0.26 / 0.26$} \\
\hline
\end{tabular}

Table 2-Input data from the IFU model for modeling organic pores in organic matter.
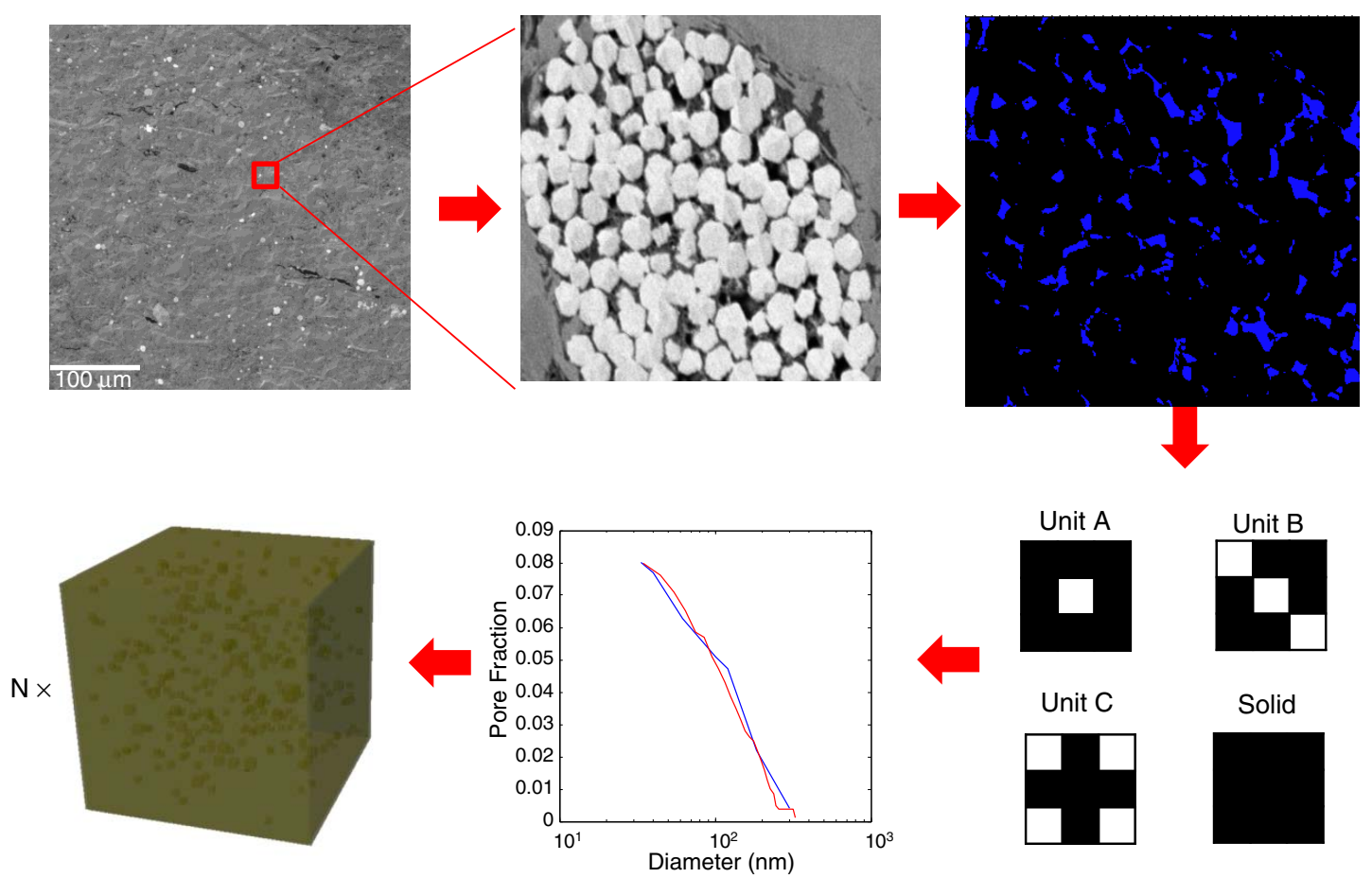

Fig. 5-Modeling process of organic pores in pyrites.

Organic Bulks in Organic Matter. Similar to the building process for the 3D-IFU model for organic pores, we extracted organic bulks using SEM images at an appropriate scale. After segmentation and binarization, we obtained the bulk-size-distribution curve of the real sample. Then, the fractal parameters were adjusted to narrow the difference between the fractal-model bulk-size-distribution curve and the real sample curve. Finally, a 3D-IFU model for organic bulks was built with the adjusted fractal parameters. When organic bulks were scattered into the final 3D IFM, each bulk was regarded as a porous medium with organic pores scattered in it. Each organic bulk was a 3D-IFU model for the organic pores in organic matter.

Inorganic Pores and Slits. Inorganic pores and slits also affect apparent gas permeability. For inorganic pores, a 3D IFU was built using SEM images that contain inorganic pores. The SEM images are shown in Fig. 6, with the area larger than its RES. The 3D-IFU building method is similar to the process discussed previously. Finally, the pores were scattered in the 3D-IFM space. The distribution 
of slits presented relatively strong heterogeneity. The slits were observed and measured at different scales in the scalable SEM images. The main parameters were the distribution and aperture of slits. We determined the statistical parameters of inorganic slits by calculating the distribution density of inorganic slits in a large area and their average length and aperture in a magnified image. The slits were then scattered in the final 3D IFM.

\begin{tabular}{|c|c|c|c|c|c|c|c|c|c|c|c|c|}
\hline & \multicolumn{3}{|c|}{ Sample 1} & \multicolumn{3}{|c|}{ Sample 2} & \multicolumn{3}{|c|}{ Sample 3} & \multicolumn{3}{|c|}{ Sample 4} \\
\hline & Unit A & Unit B & Unit C & Unit A & Unit B & Unit C & Unit A & Unit B & Unit C & Unit A & Unit B & Unit C \\
\hline$D_{F}$ & 1.89 & 1.77 & 1.26 & 1.89 & 1.77 & 1.63 & 1.89 & 1.63 & 1.46 & 1.89 & 1.77 & 1.63 \\
\hline$n$ unit & 1 & 3 & 15 & 1 & 2 & 65 & 1 & 4 & 10 & 1 & 2 & 8 \\
\hline$n$ pore $(i=1)$ & 1 & 2 & 5 & 1 & 2 & 3 & 1 & 3 & 4 & 1 & 2 & 3 \\
\hline$\lambda_{\max }(\mathrm{nm})$ & 240 & 150 & 85 & 140 & 83 & 50 & 300 & 185 & 120 & 260 & 190 & 115 \\
\hline Iteration & 2 & 2 & 2 & 3 & 2 & 2 & 3 & 2 & 2 & 3 & 2 & 2 \\
\hline$\lambda_{\min }(\mathrm{nm})$ & 80 & 50 & 28.33 & 15.56 & 27.67 & 16.67 & 33.33 & 61.66 & 40 & 28.89 & 63.33 & 38.33 \\
\hline Nolid & 0 & 0 & 0 & 0 & 0 & 0 & 0 & 0 & 0 & 0 & 0 & 0 \\
\hline $\begin{array}{l}\text { Total surface } \\
\qquad\left(\mu \mathrm{m}^{2}\right)\end{array}$ & \multicolumn{3}{|c|}{37.23} & \multicolumn{3}{|c|}{10.21} & \multicolumn{3}{|c|}{22.75} & \multicolumn{3}{|c|}{8.94} \\
\hline$\varepsilon_{S E M} / \varepsilon_{I F U}$ & \multicolumn{3}{|c|}{$0.11 / 0.11$} & \multicolumn{3}{|c|}{$0.07 / 0.07$} & \multicolumn{3}{|c|}{$0.08 / 0.08$} & \multicolumn{3}{|c|}{$0.10 / 0.11$} \\
\hline
\end{tabular}

Table 3-Input data from the IFU model for modeling organic pores in pyrites.
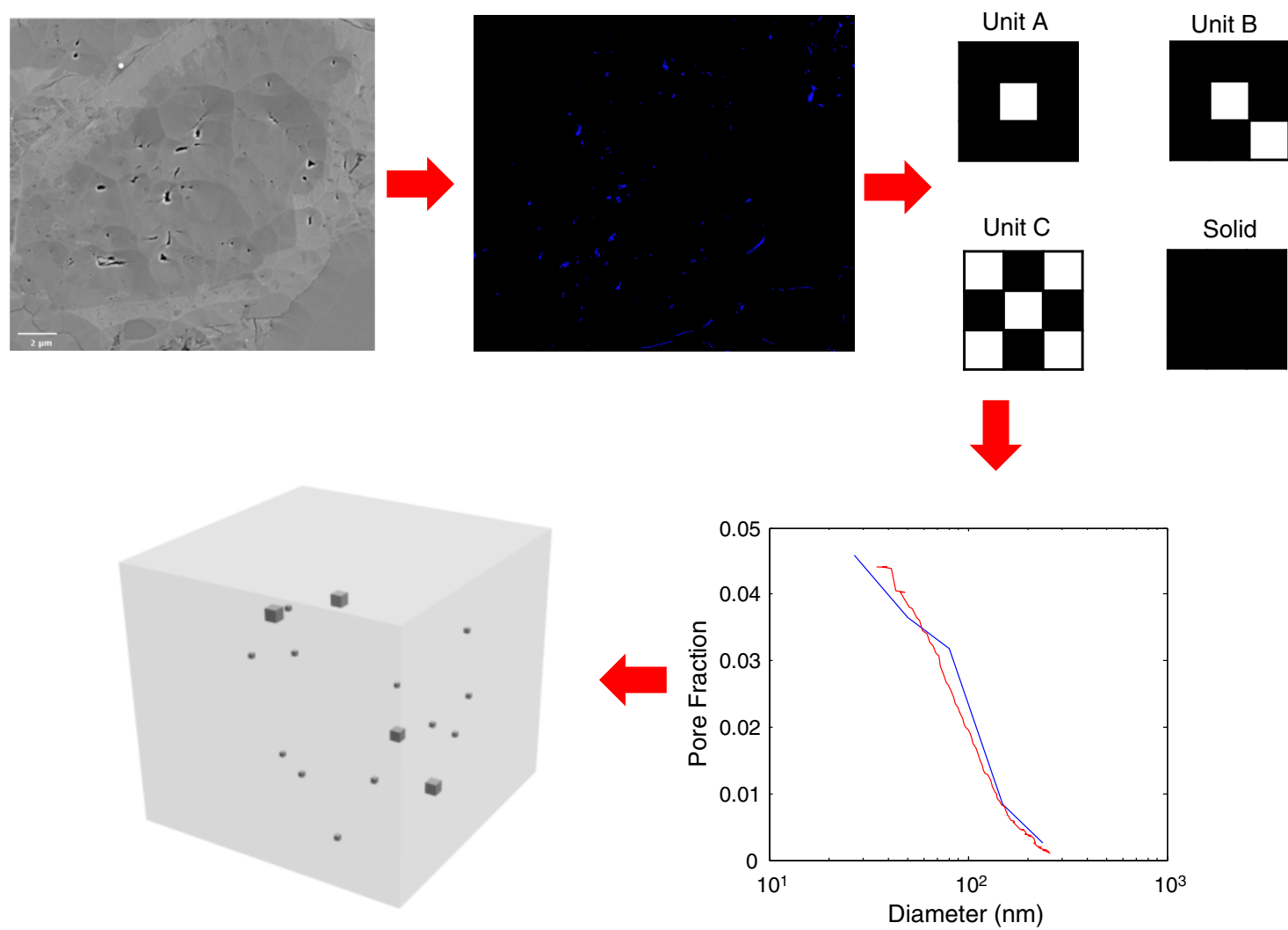

Fig. 6-Modeling process of inorganic pores.

Model Matrix. The matrix of this model was derived from the laboratory-test results. The matrix for shale is composed of several minerals, including quartz, feldspars, orthose, calcite, and clay minerals, which always include illite, chlorite, and montmorillonite. Therefore, the permeability of the matrix can be regarded as the arithmetic mean of the permeability of each component and can be expressed as

$$
k_{\text {matrix }}=\phi_{1} k_{1}+\phi_{2} k_{2}+\ldots+\phi_{n} k_{n}
$$

where $\phi_{i}$ is the percentage of each component and $k_{i}$ is the permeability of each component. For Samples 1 and 2, the percentages of key components were determined through energy-dispersive spectroscopy: $\phi_{1, \text { illite }}=0.36, \phi_{2, \text { orthose }}=0.18, \phi_{3, \text { quartz }}=0.18$, and $\phi_{4, \text { other }}=0.28$. For Samples 3 and $4, \phi_{1, \text { albite }}=0.76, \phi_{2, \text { illite }}=0.04, \phi_{3, \text { orthoclase }}=0.04$, and $\phi_{4, \text { other }}=0.16$.

Building IFM. Basic 3D-IFU models for the organic pores in organic matter and pyrites, inorganic pores, organic-matter bulks, and slits were calculated in section Building the IFM. In the current subsection, these 3D-IFU models are intermingled to build a 3D IFM. 
The detailed building process is shown in Fig. 7. The information for each component came from the scalable SEM images. The content of organic matter was measured to control the threshold value. At a scale of $40 \mu \mathrm{m}$ (shown in Fig. 3), the distribution of organic-matter bulks was easily measured, and the IFU model for organic matter was built. These organic-matter bulks are regarded as porous media in the final IFM because each organic-matter bulk contains abundant organic pores. We magnified the SEM images and observed organic pores in organic matter, organic pores in pyrite, and inorganic pores in the matrix. The fractal information of each was derived, and a 3D IFU was built for each of them. The 3D-IFU model for organic pores in organic matter is scattered in the 3D-IFU model for organic-matter bulks. The organic pores in pyrites are scattered in the 3D pyrite model using the 3D-IFU method, and the distribution density and average diameter of pyrites can be observed in the SEM images at a scale of $40 \mu \mathrm{m}$. The 3D-IFU model for inorganic pores in the matrix is directly scattered in the 3D IFM. We obtained the statistical parameters of slits by calculating their distribution density, average length, and aperture. Finally, the 3D IFM was obtained by mixing these components. The final pore-size distribution also followed the statistical data of the final SEM image. For example, SEM Image 3 has 82 pyrites. The aperture of slits varied from 20 to $85 \mathrm{~nm}$, the average aperture was $40 \mathrm{~nm}$, the average length was $5.18 \mu \mathrm{m}$, and the average distribution density was 50.3 lines $/ 10000 \mu \mathrm{m}^{2}$. We obtained a 3D IFM, as shown in the right part of Fig. 7 . The parameters for other samples are derived in the same manner. Permeability was derived from these fractal parameters. The equivalent gas permeability of the 3D IFM was determined with the PCC method.

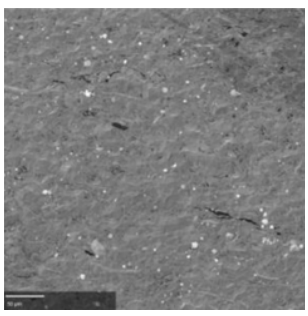

SEM image
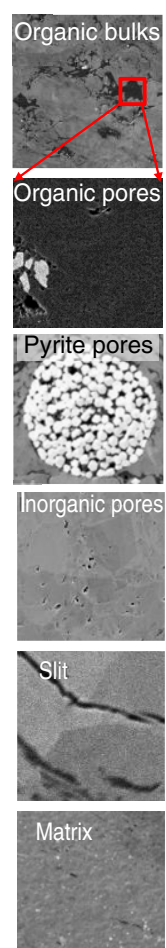

IFU model

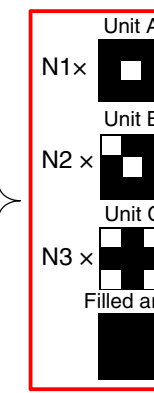

Statistical analysis

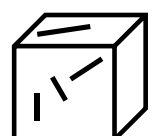

Rock-mineralogy analysis

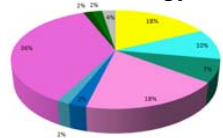

3D-IFM model

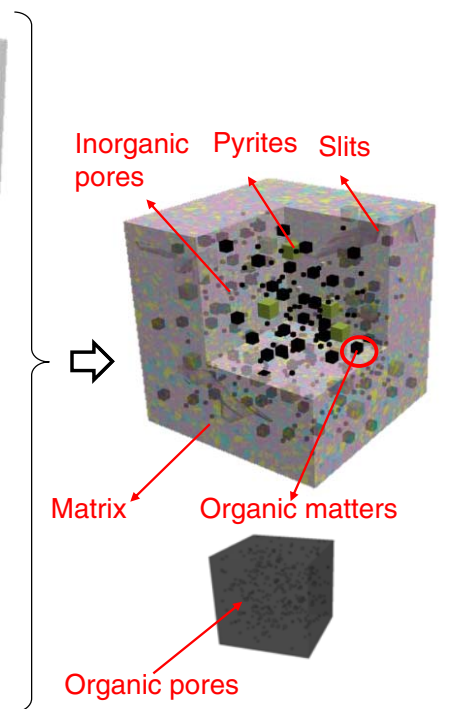

Fig. 7-IFM procedure to calculate permeability.

Apparent Gas Permeability. With all the components discussed previously, we listed the equivalent diameters of the components in a descending order in representative elementary volume (REV). The components were organic-matter bulks, pyrites, inorganic pores, and slits. According to the basic theory of the PCC method, apparent gas permeability can be expressed as

$$
k_{3 \mathrm{D}-\mathrm{IFM}}=\sum_{i=1}^{m}\left(32 \frac{\lambda_{i}^{2} N_{i}}{A} \frac{\lambda_{i}^{2} N_{i}}{A}+2 \times 32 \frac{\lambda_{1}^{2} N_{1}}{A} \frac{\lambda_{i}^{2} N_{i}}{A}+\ldots 2 \times 32 \frac{\lambda_{i-1}^{2} N_{i-1}}{A} \frac{\lambda_{i}^{2} N_{i}}{A}\right) \times C_{i} k_{i}\left(p_{\text {avg }}\right)+k_{\text {matrix }} .
$$

In the descending order of components, the blocks for each level are different. For organic-matter bulks and pyrites, the blocks represent porous media. The permeability term of the block itself is made up of several levels of pores, with IFU permeability. Shape factor $C_{i}$ here represents the shape of bulks. If we consider the expression of Eq. 15 and suggest the block is made up of IFUs with $n$th iteration level, the permeability item $k_{i}\left(p_{\text {avg }}\right)$ is expressed as

$$
k_{i}\left(p_{\text {avg }}\right)=k_{\text {sum- } n D}\left(n, p_{\text {avg }}\right) .
$$

For inorganic pores, shape factor $C=C_{\text {inorg }}$, and permeability item $k_{i}\left(p_{\text {avg }}\right)$ can be expressed as

$$
k_{i}\left(p_{\text {avg }}\right)=k_{\text {inorg- } n D}(n)=\left\{\frac{\lambda_{i} \mu M}{3 \times 10^{3} R T \rho_{\text {avg }}}\left(\frac{8 R T}{\pi M}\right)^{0.5}+\frac{\lambda_{i}^{2}}{32}\left[1+\left(\frac{8 \pi R T}{M}\right)^{0.5} \frac{2 \mu}{p_{\text {avg }} \lambda_{i}}\left(\frac{2}{\alpha}-1\right)\right]\right\} .
$$

For slits, shape factor $C=C_{\text {slit }}$, and permeability item $k_{i}\left(p_{\text {avg }}\right)$ can be expressed as

$$
k_{i}\left(p_{\text {avg }}\right)=k_{\text {slit }-n D}(n)=\left\{\frac{\lambda_{i} \mu M}{3 \times 10^{3} R T \rho_{\text {avg }}}\left(\frac{8 R T}{\pi M}\right)^{0.5}+\frac{\lambda_{i}^{2}}{32}\left[1+\left(\frac{8 \pi R T}{M}\right)^{0.5} \frac{2 \mu}{p_{\text {avg }} \lambda_{i}}\left(\frac{2}{\alpha}-1\right)\right]\right\} .
$$


For the matrix,

$k_{\text {matrix }}=\phi_{1} k_{1}+\phi_{2} k_{2}+\ldots \phi_{n} k_{n}$

\section{Validation and Improvement}

Model Validation. The 3D IFM was verified from two aspects. First, the scale of 3D IFMs should have reached the scale of REV. In a valid modeling process, the average grayscale should remain stable with the increase in the research area, shown in Fig. 8. As described, 3D IFM is made up of several components, including organic pores in organic matter and in pyrites, inorganic pores, slits, and the matrix. Similar to Fig. 4, apparent gas permeability represents the permeability of the model in unit area. When the restricted research volume (or subvolume) doubles or triples in size, pore distribution and porosity remain the same. The original points to be enlarged were selected from eight corners, the enlarged subvolumes were named Sub1 through Sub8 in Fig. 8. The result showed that when the research area was larger than 500 pixels on the edge $(25.33 \mu \mathrm{m}$ at length), the average grayscale from different directions was stable. Meanwhile, our 3D-IFM edge reached $400 \mu \mathrm{m}$, which means that the IFM area can be regarded as an REV of the upscaled image.

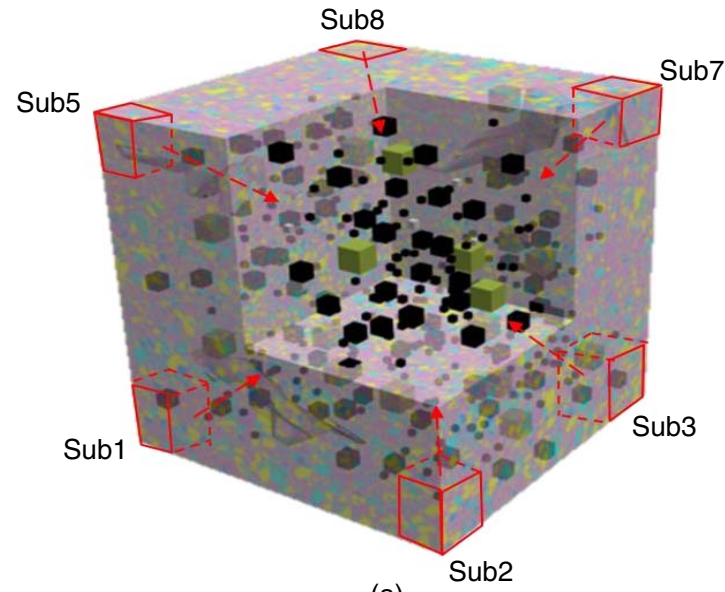

(a)

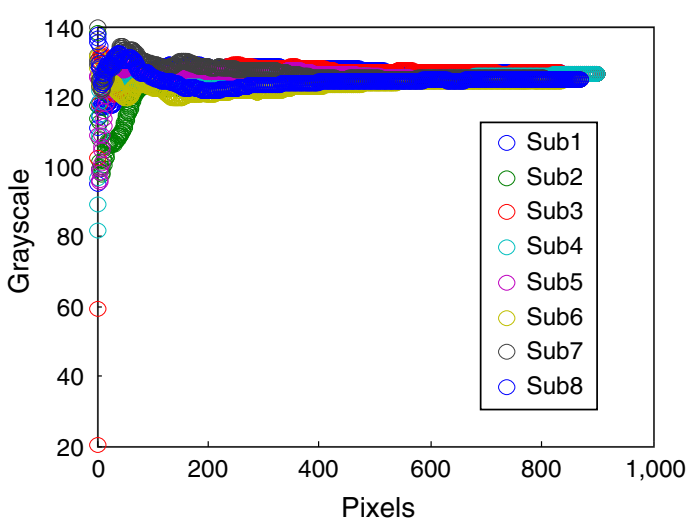

(b)

Fig. 8-Schematic of 3D IFM.

We also conducted an experimental verification to validate this model. The apparent permeability of the 3D IFM was derived by introducing experimental conditions and fractal parameters using the SEM images of samples. The experimental and 3D-IFM calculation results are shown in Table 4. The model results are generally close to the experimental data, which verify our 3D-IFU model.

\begin{tabular}{lccc} 
& $K_{\text {exp_avg }}(\mathrm{nd})$ & K $_{\text {3D-IFM }}(\mathrm{nd})$ & Error \\
\hline Sample 1 & $2,521.7$ & $1,878.27$ & $25.51 \%$ \\
Sample 2 & 39.5 & 51.13 & $29.44 \%$ \\
Sample 3 & 91.36 & 99.17 & $8.55 \%$ \\
Sample 4 & 91.36 & 103.23 & $13.00 \%$ \\
\hline
\end{tabular}

Table 4-Comparison of experimental and IFM permeability data.

Advantages of 3D IFM Compared With Quasi-3D IFM. The 3D IFM in this work showed two advantages over the quasi-3D IFM discussed in our previous work: improved accuracy and wider application area.

The most important advantage is improved accuracy. As indicated by the calculation results, the 3D IFM more-effectively calculated the permeability of organic-rich shale on the basis of 2D SEM images than did the quasi-3D IFM. The detailed building process of this model was presented by Li et al. (2017). The comparison of the final permeability solutions for the quasi-3D and 3D models is shown in Table 5. The revised 3D IFM using the pore-connective-calculation method showed slightly improved accuracy in permeability calculation for organic-rich shale.

\begin{tabular}{c|ccc|cc} 
& $K_{\text {quasi-3D-IFM }}(\mathrm{nd})$ & Kexp_avg $_{\text {exd }}$ & $K_{\text {3D-IFM }}(\mathrm{nd})$ & Error $_{\text {quasi-3D IFM }}$ & Error $_{\text {3D-IFM }}$ \\
\hline Sample 3 & 105.70 & 91.36 & 99.17 & $13.57 \%$ & $8.55 \%$ \\
Sample 4 & 111.88 & 91.36 & 103.23 & $18.34 \%$ & $13.00 \%$ \\
\hline
\end{tabular}

Table 5-Comparison of quasi-3D-IFM and 3D-IFM results.

In addition, the 3D IFM in this work showed a broader application than the quasi-3D IFM discussed in our previous work. The 3D IFM considers the main components, such as organic pores in organic matter and in pyrites, organic bulks, inorganic pores, slits, and the matrix. The application area of the 3D-IFM calculation method is much wider than that of the quasi-3D IFM. When fractal tortuosity can be used for the representation of pore space, quasi-3D IFM is preferred. Meanwhile, under certain circumstances, such as when the pore space of shale is highly complicated, representing the pore space using only tortuosity is insufficient. The PCC method is suitable for this case. 
To summarize, the 3D-IFM method derived in this study is a good method for rapidly evaluating the permeability of organic-rich shale. The method, which is derived from fractal theory, can significantly shorten the calculation time and provides a promising means of rapidly evaluating apparent gas permeability in shale.

\section{Conclusions}

A rapid-permeability-evaluation method for organic-rich shale that is derived from 3D IFM was introduced. First, an upscaled 3D IFM for organic-rich shale was successfully built using high-resolution SEM images of shale samples. This 3D IFM considers organic pores in organic matter and in pyrites, inorganic pores, and slits. Second, an improved PCC method was introduced to calculate the gas permeability of the 3D IFM. Finally, the accuracy and reliability of this model were examined depending on the experimental results, which indicated that this method is useful for rapid permeability evaluation.

The findings show that the proposed rebuilding method for organic-rich shale is valid and useful. The IFM takes advantage of the detailed information at the micro/nanoscale and the distribution of components in a large observation area. The introduction of the fractal method greatly simplified the expression of pore space, which provided a solid foundation for percolation calculation. Non-Darcy effects in gas flow should be considered in micro/nanopores under low pressures. In addition, the revised 3D IFM using the PCC method showed slightly improved accuracy in permeability calculation for organic-rich shale. The revised 3D IFM also has a wider application than the original quasi-3D IFM. This rapid-permeability-evaluation method can significantly simplify apparent-gaspermeability calculation and shows potential for use in future applications.

\section{Nomenclature}

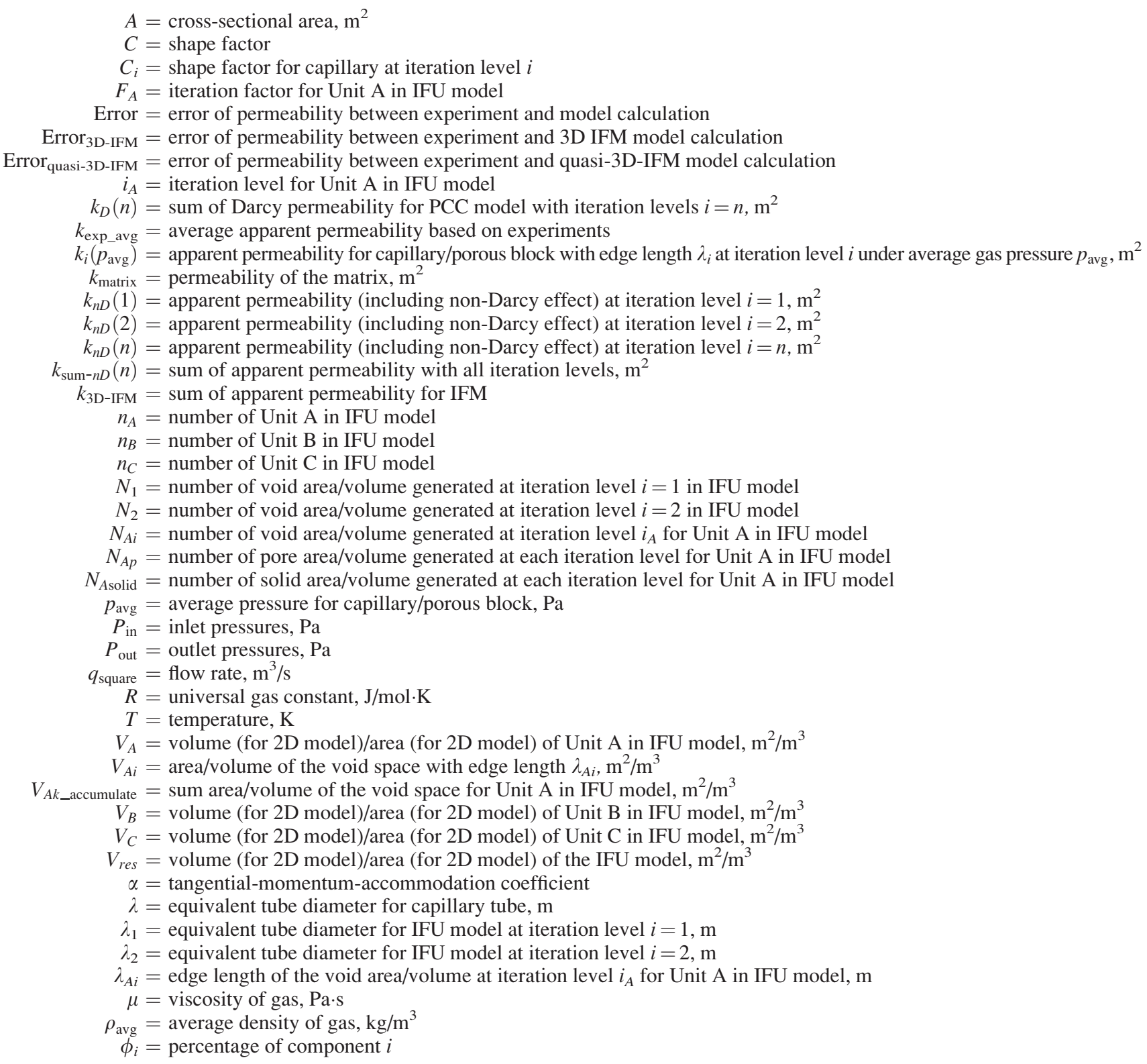

\section{Acknowledgments}

This work is supported by the National Natural Science Foundation of China (Grant No. 41690132), the Strategic Priority Research Program of the Chinese Academy of Sciences (Grant No. XDA14010101), the Major National Science and Technology Special Program of China (Grant No. 2017ZX05037-001), the National Natural Science Foundation of China (Grant No. 41574129), and the Strategic Priority Research Program of the Chinese Academy of Sciences (Grant No. XDB10020302). 


\section{References}

Adler, P. M. 1996. Transports in Fractal Porous Media. J. Hydrol. 187 (1-2): 195-213. https://doi.org/10.1016/s0022-1694(96)03096-x.

Adler, P. M. and Thovert, J.-F. 1998. Real Porous Media: Local Geometry and Macroscopic Properties. Appl. Mech. Rev. 51 (9): 537-585. https:// doi.org/10.1115/1.3099022.

Balankin, A. S. 2013. Stresses and Strains in a Deformable Fractal Medium and its Fractal Continuum Model. Phys. Lett. A. 377 (38): $2535-2541$. https://doi.org/10.1016/j.physleta.2013.07.029.

Balankin, A. S. 2015a. A Continuum Framework for Mechanics of Fractal Materials I: From Fractional Space to Continuum With Fractal Metric. Eur. Phys. J. B. 88 (4): 90. https://doi.org/10.1140/epjb/e2015-60189-y.

Balankin, A. S. 2015b. A Continuum Framework for Mechanics of Fractal Materials II: Elastic Stress Fields Ahead of Cracks in a Fractal Medium. Eur. Phys. J. B. 88 (4): 91. https://doi.org/10.1140/epjb/e2015-50703-8.

Balankin, A. S. and Elizarraraz, B. E. 2012a. Hydrodynamics of Fractal Continuum Flow. Phys. Rev. E. 85 (22): 025302. https://doi.org/10.1103/ PhysRevE.85.025302.

Balankin, A. S. and Elizarraraz, B. E. 2012b. Map of Fluid Flow in Fractal Porous Medium into Fractal Continuum Flow. Phys. Rev. E. 85 (5): 056314. https://doi.org/10.1103/PhysRevE.85.056314.

Bryant, S. and Blunt, M. 1992. Prediction of Relative Permeability in Simple Porous Media. Phys. Rev. A. 46 (4): 2004-2011. https://doi.org/10.1103/ physreva.46.2004.

Bryant, S., Cade, C., and Mellor, D. 1993. Permeability Prediction From Geologic Models. AAPG Bull. 77 (8): 1338-1350. https://doi.org/10.1306/ bdff8e 84-1718-11d7-8645000102c1865d.

Cai, J.-C. 2014. A Fractal Approach to Low Velocity Non-Darcy Flow in a Low Permeability Porous Medium. Chinese Phys. B. 23 (4): 044701. https:// doi.org/10.1088/1674-1056/23/4/044701.

Cai, J.-C., Perfect, E., Cheng, C.-L. et al. 2014. Generalized Modeling of Spontaneous Imbibition Based on Hagen-Poiseuille Flow in Tortuous Capillaries with Variably Shaped Apertures. Langmuir 30 (18): 5142-5151. https://doi.org/10.1021/la5007204.

Cai, J.-C., Yu, B., Zou, M. et al. 2010. Fractal Characterization of Spontaneous Co-Current Imbibition in Porous Media. Energy Fuels 24 (3): 1860-1867. https://doi.org/10.1021/ef901413p.

Cihan, A., Birkholzer, J., Illangasekare, T. H. et al. 2014. A Modeling Approach to Represent Hysteresis in Capillary Pressure-Saturation Relationship Based on Fluid Connectivity in Void Space. Water Resour. Res. 50 (1): 119-131. https://doi.org/10.1002/2013WR014280.

Cihan, A., Birkholzer, J., Trevisan, L. et al. 2017. Investigation of Representing Hysteresis in Macroscopic Models of Two-Phase Flow in Porous Media Using Intermediate Scale Experimental Data. Water Resour. Res. 53 (1): 199-221. https://doi.org/10.1002/2016WR019449

Cihan, A., Perfect, E., and Tyner, J. S. 2008. Water Retention Models for Scale-Variant and Scale-Invariant Drainage of Mass Prefractal Porous Media. Vadose Zone J. 7 (1): 396. https://doi.org/10.2136/vzj2007.0062er.

Cihan, A., Sukop, M. C., Tyner, J. S. et al. 2009a. Analytical Predictions and Lattice Boltzmann Simulations of Intrinsic Permeability for Mass Fractal Porous Media. Vadose Zone J. 8 (1): 187-196. https://doi.org/10.2136/vzj2008.0003.

Cihan, A., Tyner, J. S., and Perfect, E. 2009b. Predicting Relative Permeability From Water Retention: A Direct Approach Based on Fractal Geometry. Water Resour. Res. 45 (4): W04404. https://doi.org/10.1029/2008WR007038.

Cooper, S. M., Cruden, B. A., Meyyappan, M. et al. 2004. Gas Transport Characteristics Through a Carbon Nanotubule: Nano Letters 4 (2): $377-381$. https://doi.org/10.1021/n10350682.

Fatt, I. 1956. The Network Model of Porous Media. SPE-574-G.

Feng, Y. and Gray, K. E. 2017. Modeling Lost Circulation Through Drilling-Induced Fractures. SPE J. 23 (1): 1-19. SPE-187945-PA. https://doi.org/ 10.2118/187945-PA.

Fischer, U. and Celia, M. A. 1999. Prediction of Relative and Absolute Permeabilities for Gas and Water From Soil Water Retention Curves Using a Pore-Scale Network Model. Water Resour. Res. 35 (4): 1089-1100. https://doi.org/10.1029/1998wr900048.

Javadpour, F. 2009. Nanopores and Apparent Permeability of Gas Flow in Mudrocks (Shales and Siltstone). J Can Pet. Technol 48 (8): 16-21. PETSOC09-08-16-DA. https://doi.org/10.2118/09-08-16-DA.

Javadpour. F. and Ettehadtavakkol, A. 2015. Fundamentals of Gas Shale Reservoirs. In Gas Transport Processes in Shale, ed. Rezaee R., Chap. 11, 245-266. Hoboken, New Jersey: John Wiley \& Sons.

Javadpour, F., Fisher, D., and Unsworth, M. 2007. Nanoscale Gas Flow in Shale Gas Sediments. J Can Pet Technol 46 (10): 55-61. PETSOC-07-10-06. https://doi.org/10.2118/07-10-06.

Jiang, W., Lin, M., Yi, Z. et al. 2017. Parameter Determination Using 3D FIB-SEM Images for Development of Effective Model of Shale Gas Flow in Nanoscale Pore Clusters. Transport Porous Med. 117 (1): 5-25. https://doi.org/10.1007/s11242-016-0817-5.

Lee, A. L., Gonzalez, M. H., and Eakin, B. E. 1966. The Viscosity of Natural Gases. J Pet Technol 18 (8): 997-1000. SPE-1340-PA. https://doi.org/ 10.2118/1340-PA.

Li, C., Lin, M., Ji, L. et al. 2017. Investigation of Intermingled Fractal Model for Organic-Rich Shale. Energy Fuels 31 (9): 8896-8909. https://doi.org/ 10.1021/acs.energyfuels.7b00834.

Lin, D., Wang, J., Yuan, B. et al. 2017. Review on Gas Flow and Recovery in Unconventional Porous Rocks. Adv. Geo-Energy Res. 1 (1): 39-53. https:// doi.org/10.26804/ager.2017.01.04.

Lu, T., Li, Z., Lai, F. et al. 2018. The Effect of Flow Resistance on Water Saturation Profile for Transient Two-Phase Flow in Fractal Porous Media. Adv. Geo-Energy Res. 2 (1): 63-71. https://doi.org/10.26804/ager.2018.01.06.

Marshall, T. J. 1958. A Relation Between Permeability and Size Distribution of Pores. J. Soil Sci. 9 (1): 1-8. https://doi.org/10.1111/j.13652389.1958.tb01892.x.

Niu, C., Hao, Y.-Z., Li, D. et al. 2014. Second-Order Gas-Permeability Correlation of Shale During Slip Flow. SPE J. 19 (5):786-792. SPE-168226-PA. https://doi.org/10.2118/168226-PA.

Perfect, E. 2005. Modeling the Primary Drainage Curve of Prefractal Porous Media. Vadose Zone J. 4 (4): 959-966. https://doi.org/10.2136/ vzj2005.0012.

Perfect, E., Gentry, R. W., Sukop, M. C. et al. 2006. Multifractal Sierpinski Carpets: Theory and Application to Upscaling Effective Saturated Hydraulic Conductivity. Geoderma 134 (3-4): 240-252. https://doi.org/10.1016/j.geoderma.2006.03.001.

Pia, G. 2016. Fluid Flow in Complex Porous Media: Experimental Data and IFU Model Predictions for Water Vapour Permeability. J. Nat. Gas Sci. Eng. 35A (September): 283-290. https://doi.org/10.1016/j.jngse.2016.08.053.

Pia, G. and Sanna, U. 2013a. A Geometrical Fractal Model for the Porosity and Thermal Conductivity of Insulating Concrete. Constr. Build. Mater. 44 (July): 551-556. https://doi.org/10.1016/j.conbuildmat.2013.03.049.

Pia, G. and Sanna, U. 2013b. Intermingled Fractal Units Model and Electrical Equivalence Fractal Approach for Prediction of Thermal Conductivity of Porous Materials. Appl. Therm. Eng. 61 (2): 186-192. https://doi.org/10.1016/j.applthermaleng.2013.07.031. 
Pia, G. and Sanna, U. 2014. An Intermingled Fractal Units Model and Method to Predict Permeability in Porous Rock. Int. J. Eng. Sci. 75 (February): 31-39. https://doi.org/10.1016/j.ijengsci.2013.11.002.

Pia, G., Siligardi, C., Casnedi, L. et al. 2016. Pore Size Distribution and Porosity Influence on Sorptivity of Ceramic Tiles: From Experimental Data to Fractal Modelling. Ceram. Int. 42 (8): 9583-9590. https://doi.org/10.1016/j.ceramint.2016.03.041.

Roy, S., Raju, R., Chuang, H. F. et al. 2003. Modeling Gas Flow Through Microchannels and Nanopores. J. Appl. Phys. 93 (8): 4870-4879. https:// doi.org/10.1063/1.1559936.

Shabro, V., Javadpour, F., and Torres-Verdín, C. 2009. A Generalized Finite-Difference Diffusive-Advective (FDDA) Model for Gas Flow in Microand Nano-Porous Media. World J. Eng. 6: 7-15.

Shabro, V., Kelly, S., Torres-Verdín, C. et al. 2014. Pore-Scale Modeling of Electrical Resistivity and Permeability in FIB-SEM Images of Organic Mudrock. Geophysics 79 (5): D289-D299. https://doi.org/10.1190/geo2014-0141.1.

Shabro, V., Torres-Verdin, C., and Sepehrnoori, K. 2012. Forecasting Gas Production in Organic Shale with the Combined Numerical Simulation of Gas Diffusion in Kerogen, Langmuir Desorption From Kerogen Surfaces, and Advection in Nanopores. Presented at the SPE Annual Technical Conference and Exhibition, San Antonio, Texas, 8-10 October. SPE-159250-MS. https://doi.org/10.2118/159250-MS.

Shou, D., Fan, J., and Ding, F. 2010. A Difference-Fractal Model for the Permeability of Fibrous Porous Media. Phys. Lett. A 374 (10): $1201-1204$. https://doi.org/10.1016/j.physleta.2010.01.002.

Thompson, A. H., Katz, A. J., and Krohn, C. E. 1987. The Microgeometry and Transport Properties of Sedimentary Rocks. Adv. Phys. 36 (5): 625-694. https://doi.org/10.1080/00018738700101062.

Thovert, J. F., Wary, F., and Adler, P. M. 1990. Thermal Conductivity of Random Media and Regular Fractals. J. Appl. Phys. 68 (8): 3872-3883. https:// doi.org/10.1063/1.346274.

Veltzke, T. and Thöming, J. 2012. An Analytically Predictive Model for Moderately Rarefied Gas Flow. J. Fluid Mech. 698 (10 May): $406-422$. https:// doi.org/10.1017/jfm.2012.98.

Wei, W., Cai, J., Hu, X. et al. 2015. A Numerical Study on Fractal Dimensions of Current Streamlines in Two-Dimensional and Three-Dimensional Pore Fractal Models of Porous Media. Fractals 23 (1):1540012. https://doi.org/10.1142/s0218348x15400125.

Wu, K., Chen, Z., Xu, J. et al. 2016a. A Universal Model of Water Flow Through Nanopores in Unconventional Reservoirs: Relationships Between Slip, Wettability and Viscosity. Presented at the SPE Annual Technical Conference and Exhibition, Dubai, 26-28 September. SPE-181542-MS. https:// doi.org/10.2118/181543-MS.

Wu, K., Li, X., Guo, C. et al. 2016b. A Unified Model for Gas Transfer in Nanopores of Shale-Gas Reservoirs: Coupling Pore Diffusion and Surface Diffusion. SPE J. 21 (5): 1583-1611. SPE-2014-1921039-PA. https://doi.org/10.2118/2014-1921039-PA.

Xiao, B., Chen, H., Xiao, S. et al. 2017. Research on Relative Permeability of Nanofibers With Capillary Pressure Effect by Means of Fractal-Monte Carlo Technique. J. Nanosci. Nanotechnol. 17 (9): 6811-6817. https://doi.org/10.1166/jnn.2017.14502.

Xu, P. and Yu, B. 2008. M. Developing a New Form of Permeability and Kozeny-Carman Constant for Homogeneous Porous Media by Means of Fractal Geometry. Adv. Water Resour. 31 (1): 74-81. https://doi.org/10.1016/j.advwatres.2007.06.003.

Yu, B. M. 2005. Fractal Character for Tortuous Streamtubes in Porous Media. Chin. Phys. Lett. 22 (1): 158-160. https://doi.org/10.1088/0256-307x/22/ $1 / 045$.

Yu, B. M. and Li, J. H. 2001. Some Fractal Characters of Porous Media. Fractals 9 (3): 365-372. https://doi.org/10.1142/s0218348x01000804.

Yu, B. M., Lee, L. J. and Cao, H. Q. 2002. A Fractal In-Plane Permeability Model for Fabrics. Polym. Compos. 23 (2): 201-221. https://doi.org/10.1002/ pc.10426.

Yuan, B., Zheng, D., Moghanloo, R.G. et al. 2017. A. Novel Integrated Workflow for Evaluation, Optimization, and Production Predication in Shale Plays. Int. J. Coal Geol. 180 (1 July): 18-28. https://doi.org/10.1016/j.coal.2017.04.014.

Caoxiong Li is a PhD degree student in the Institute of Mechanics at the Chinese Academy of Sciences. His interests include shale-gas-flow mechanism, fractals, and spontaneous imbibition. Li has authored or coauthored more than seven technical papers related to those topics. He holds a bachelor's degree in petroleum engineering from China University of Petroleum, East China, and a master's degree in petroleum engineering from China University of Petroleum, Beijing. Li serves as a technical reviewer for several professional journals and is a student member of SPE.

Mian Lin is a professor of fluid mechanics in the Institute of Mechanics at the Chinese Academy of Sciences. Her main interests include flow mechanisms in shale-gas production and rebuilding and upscaling of pore space in unconventional reservoirs. Lin has presented or published more than 20 papers on related topics.

Lili $\mathbf{J i}$ is an assistant professor in the Institute of Mechanics at the Chinese Academy of Sciences. Her main interests include rebuilding and upscaling of pore space in unconventional reservoirs and gas- and liquid-flow mechanisms for shale. Ji holds a $\mathrm{PhD}$ degree from the Institute of Mechanics at the Chinese Academy of Sciences.

Wenbin Jiang is an associate professor in the Institute of Mechanics at the Chinese Academy of Sciences. His interests include flow mechanisms in shale-gas production and rebuilding and upscaling of pore space in unconventional reservoirs. Jiang holds a master's degree from Tsinghua University, China, and a PhD degree from the Institute of Mechanics at the Chinese Academy of Sciences.

Gaohui Cao is a PhD degree student at the Chinese Academy of Sciences, with current research interests that include gas-flow mechanisms and model upscaling for shale. 